\title{
Trepostomids (Bryozoa) from the Devonian of Salair, Kuznetsky Basin, Gorny and Rudny Altai, Russia
}

\author{
OLgA P. MESENTSEVA
}

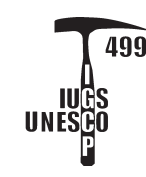

\begin{abstract}
Trepostomid bryozoa from the Devonian of the Salair-Altai region (SAR) have been investigated. Analysis of collected data has allowed the late Silurian-Devonian-Tournaisian interval to be divided into 16 stages, each represented by a specific association of trepostomids. Cyclic changes in trepostomid species composition are marked by alternations of low diversity. Four cycles can be recognized, beginning with an interval of high species diversity and ending with an interval of low species diversity. Synchronicity between cycles of species diversity and recognized transgressive-regressive cycles is evident in the SAR. Diversity peaks correspond to transgressions, whereas minimum diversity to regressions. Local cyclicity of the trepostomid diversity in the SAR does not coincide with a global bryozoan cyclicity. Species duration of Devonian trepostomids is assessed and their potential for biostratigraphical correlation within the SAR proven. $\bullet$ Key words: bryozoans, trepostomids, species association, diversity changes, Devonian, Salair-Altai region.
\end{abstract}

MesentseVA, O.P. 2008. Trepostomids (Bryozoa) from the Devonian of Salair, Kuznetsky Basin, Gorny and Rudny Altai, Russia. Bulletin of Geosciences 83(4), 449-460 (8 figures). Czech Geological Survey, Prague. ISSN 1214-1119. Manuscript received November 13, 2006; accepted in revised form February 28, 2007; issued December 31, 2008.

Olga P. Mesentseva, Kuzbass State Education Academy, Kuznetsova st. 6, Novokuznetsk, 654041, Russia; MesentsevaOP@yandex.ru

The territories of the Salair, Gorny and Rudny Altai Mountains in central Asia are now regarded as the Salair-Altai region (SAR). Until the end of the $20^{\text {th }}$ century Devonian bryozoans from the SAR, including trepostomids, were rarely investigated. Late Givetian and Frasnian examples were described in detail by Nekhoroshev (1948), Morozova (1961) and Volkova (1974), whereas Lochkovian-Pragian (Astrova \& Yaroshinskaya 1968) and Frasnian (Morozova, 1961) trepostomids were poorly investigated and Emsian-Early Givetian trepostomids were completely unstudied. Therefore it was impossible to trace changes in trepostomid specific composition during the Devonian in the region. Also, trepostomids as well as other bryozoans were not used as paleontological evidence during the construction of the stratigraphic charts for the SAR.

Within the last 20 years most part of these gaps have been filled. Yaroshinskaya studied Prrídolian-early Lochkovian trepostomids (Pushkin et al. 1990, Yaroshinskaya 1997) and Mesentseva and Udodov have described more than 60 species of Emsian-Eifelian-Early Givetian trepostomids, including 42 new species (Mesentseva 1997, 2000; Mesentseva \& Udodov 2003). The distributions of these species in several dozen sections in the SAR have been investigated. New data on Famennian trepostomids within the vicinity of the Kuznetsk Basin of Russia have also been obtained (Tolokonnikova 2006). In total, more than 150 spe- cies of Devonian trepostomids are now known from numerous localities more or less regularly distributed across the SAR territory (Fig. 1).

Special attention has been given to Devonian reference sections from the Altai-Sayan Folded Area (ASFA), exposed on the eastern slope of the Salair Ridge (near Gur'evsk town) and in the northwestern margin of the Kuznetsk Basin. The Devonian stratigraphic charts for the ASFA and the SAR were created mainly on the basis of detailed studies of these sections. In the modern version of this chart (Yolkin et al. 2005), 16 horizons (regional stages) are recognized in the Devonian (Fig. 2).

The Tom'-Chumysh, Salairka and Shanda horizons are subdivided into beds with characteristic faunas (formations, subhorizons). Based on the detailed subdivision of Devonian strata in the reference sections, trepostomid species can be placed reliably into a biostratigraphic framework. The majority of strata recognized in Salair have also been traced into Altai where they have local names. Trepostomids are very rare in Lochkovian of Salair (Tom'-Chumysh and Petz horizons) as well as in Pragian (Krekov and Maly Bachat horizons). In Salair, the early Lochkovian strata of the Tom'-Chumysh Horizon, divided into the Sukhoi and Tomskiy Zavod formations (beds with fauna), contain very few trepostomids. Therefore, the present paper deals with trepostomids from the stratigraphic 


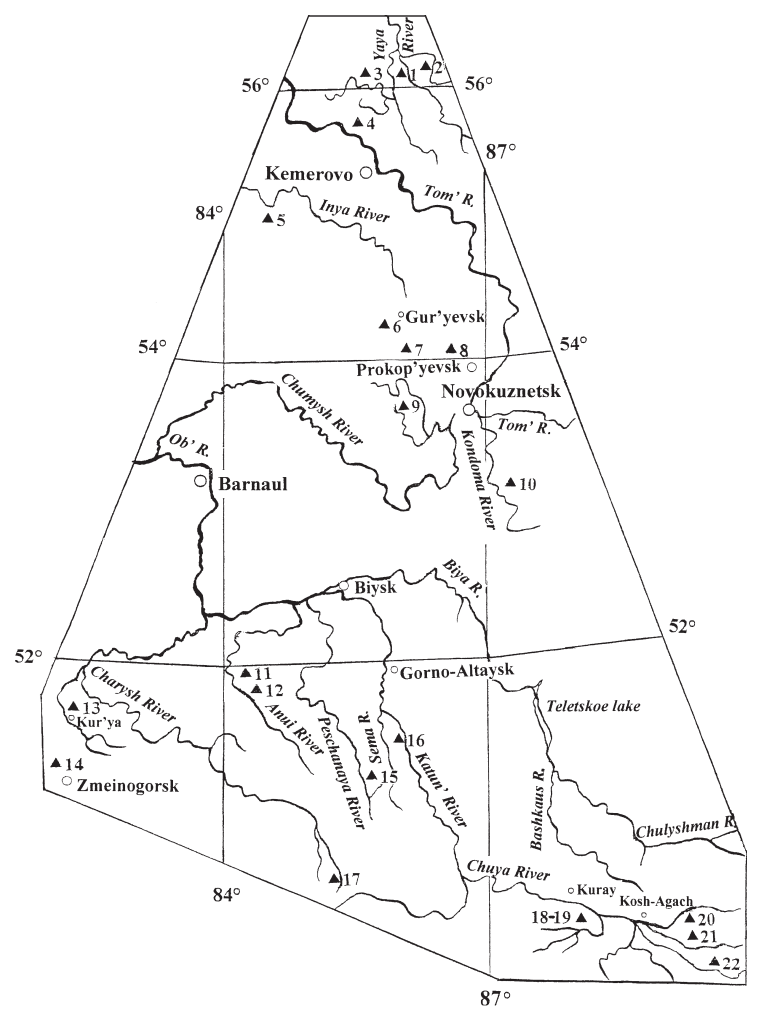

equivalents of these strata, namely the Sibirka, Remnyov and Yakushin beds from Altai.

\section{Materials and methods}

Bryozoan collections from the type sections of the Přídolian (upper Silurian), Devonian and Tournaisian (Lower Carboniferous) of the SAR have been accumulated by the author over 25 years. Using standard techniques, the bryozoans were studied in thin section under a binocular microscope. Special attention was paid to intraspecific variability, and therefore, 10 or more colonies of each species were studied. The studied collections are stored in the Paleontological Museum of the Kuzbass State Education Academy, where. In addition, previously published results of the following researchers were used: Nekhoroshev (1948, 1956), Trizna (1958), Morozova (1961), Yaroshinskaya (in Astrova \& Yaroshinskaya 1968; Pushkin et al. 1990), Volkova (1974), Udodov (2004), and Tolokonnikova (2006).

\section{Trepostomid distribution in Devonian of Salair, Kuznetsk Basin, Gorny and Rudny Altai}

The distribution of Devonian trepostomids from the SAR is summarized in below. In most cases, a rather specific trepostomid association characterizes each horizon of the regional
Figure 1. Map showing localities with Devonian trepostomids in the Salair-Altai region, Russia. 1 - Yaya; Upper Devonian (Rzonsnitskaya 1968, sine 1992). 2 - Alchedat; Middle Devonian, Upper Givetian (Rzonsnitskaya 1968; sine 1992). 3 - Mazalovsky Kitat; Middle Devonian, Upper Givetian (Rzonsnitskaya 1968; sine 1992). 4 - Kemerovo; Upper Devonian (Bakharev et al. 2004). 5 - Vassino; Upper Devonian (Rzonsnitskaya 1968; sine 1992). 6 - Gur'evsk; Lower Devonian-Middle Devonian, Eifelian (Yolkin et al. 1986, 1987; Bakharev et al. 2004). 7 - Stary Bachat; Middle Devonian, Lower Givetian (Mesentseva et al. 2007). 8 - Prokop'evsk-Kiselevsk; Middle Devonian, Lower Givetian (Mesentseva et al. 2007). 9 - Kara-Chumysh; Lower Devonian, Emsian-Middle Devonian, Eifelian (Mesentseva \& Udodov 2000). 10 - Osman; Upper Devonian (Rzonsnitskaja 1968, Udodov et al. 1987). 11 - Ganin Creek; Lower Devonian (Yolkin 1968). 12 - Sibiryachikha; Lower Devonian (Stepanov et al. 1972, Mesentseva \& Udodov 2007). 13 - Kur'ya; Lower Devonian (Bakharev et al. 2004). 14 - Zmeinogorsk; Lower Devonian, Emsian-Middle Devonian (Bakharev et al. 2004). 15 - Kuvash; Lower Devonian, Emsian (Yolkin 1968, Mesentseva \& Udodov 2007). 16 - Sema; Lower Devonian, Emsian (Mesentseva \& Udodov 2007). 17 - Mendursakon; Lower Devonian, Emsian-Middle Devonian (Mesentseva \& Udodov 2007). 18 - Daya; Lower Devonian, Emsian (Volkova 1974). 19 - Chagan-Uzun; Middle Devonian, Upper Givetian-Upper Devonian (Volkova 1974). 20 - Tabachek; Middle Devonian, Upper Givetian-Upper Devonian (Volkova 1974). 21 - Kyzyl-Shin; Middle Devonian, Upper Givetian-Upper Devonian (Volkova 1974, Gutak 2006). 22 - Bar-Burgazy; Upper Devonian, Frasnian (Volkova 1974).

stratigraphic chart. Major changes in trepostomid species usually coincide with boundaries between strata. Whereas such faunal changes usually correlate with facies changes, the fact that the material used for this study came from many distant sections means that changes in trepostomids were caused more by extinction and origination than by simple facies changes. Thus, in most cases the duration of formation for each horizon can be regarded as a stage in trepostomid evolution. In some cases major changes in trepostomid fauna coincide not with bed rather than horizon boundaries. As a result, the boundaries of the trepostomid stages (biozones) do not always coincide with the boundaries of horizons. Within the Přídolí-Early Tournaisian interval 16 stages those boundaries are interpreted as innovation boundaries. The brief characteristics of each stage are given below.

\section{Ludlow}

Trepostomid species present: Lioclema varium Astrova, 1959, Eridotrypa alternans Astrova, 1965, Amplexopora subseptosa Modzalevskaya, 1979, Discotrypa podolica (Astrova, 1965).

\section{Př́idolí}

Př́dolian stage. - Age: Přídolian (upper part of Kuimov and Cherny Anui formations). Trepostomid species present: 


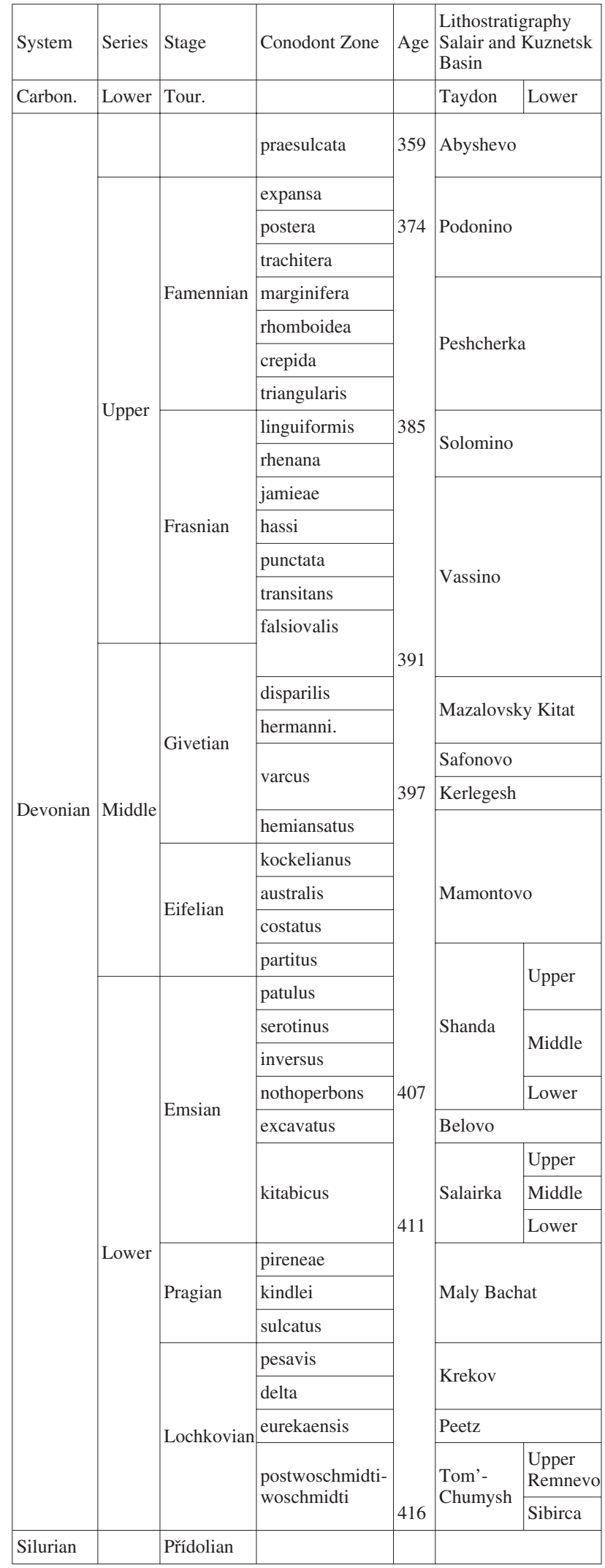

Figure 2. Regional stratigraphic chart for the Devonian of the Salair region (modified from Yolkin et al. 1997).

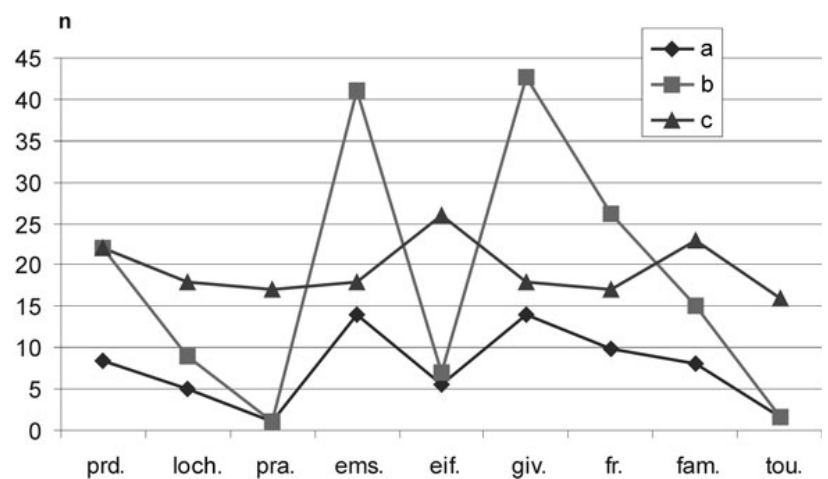

Figure 3. Dynamics of trepostomid specific and generic diversity during Př́ídolian-Devonian-Early Carboniferous. a, $\mathrm{b}$ - trepostomid diversity from the Salair-Altai region: $\mathrm{a}-$ species, $\mathrm{b}-$ genera, $\mathrm{c}-$ global generic trepostomid diversity (after Gorynova et al. 2004); n - number of species (genera).

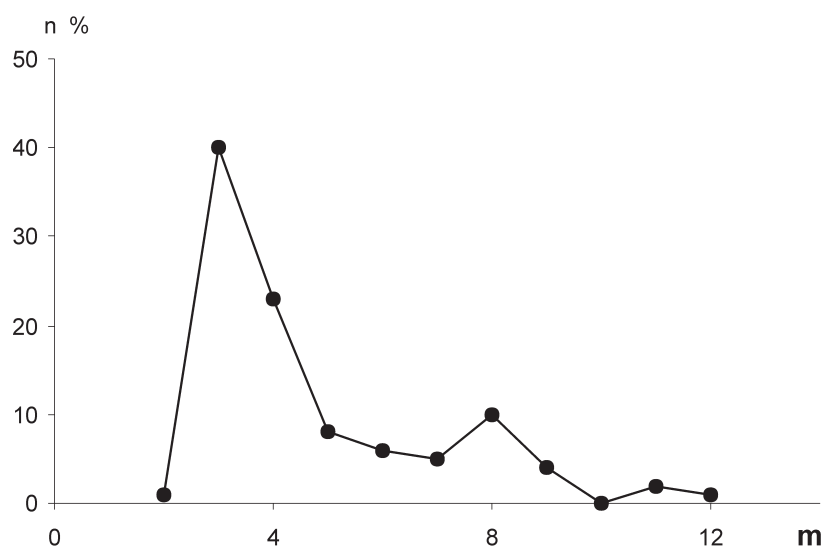

Figure 4. Ranges of Př́idolian-Devonian-Early Carboniferous trepostomids (Bryozoa) from the Salair, vicinity of the Kuznetsk Basin, Gorny and Rudny Altai. Trepostomid species lifespan was estimated on the basis of investigation of their ranges in the Altai-Sayan Folded Area sections and duration of the Silurian, Devonian and Carboniferous stages (Gradstein et al. 2004). n - species number (\%) from the total number (151 species); $\mathrm{m}$ - ranges of existence (Ma).

Lioclema varium Astrova, 1959, Eridotrypa alternans Astrova, 1965, Amplexopora subseptosa Modzalevskaya, 1979, Discotrypa podolica (Astrova, 1965), Eridotrypa callosa Morozova, 1961, Eridotrypa acifera Modzalevskaya, 1978, Eridotrypa angusta Yaroshinskaya, 1990, Hemieridotrypa altaica Kopaevich, 1968, Hemieridotrypa insolens Kopaevich, 1968, Hemieridotrypa tscherkesovae Astrova, 1965, Lioclema morozovae Yaroshinskaya, 1990, Lioclema altaica Yaroshinskaya, 1997, Lioclema praepassitabulatum Yaroshinskaya, 1990, Discotrypa infans Yaroshinskaya, 1997, Heterotrypa enormis Astrova, 1959, Lioclema gloria Astrova, 1964, Atactotoechus verus Yaroshinskaya, 1990, Eridotrypella ampla Yaroshinskaya, 1990, Lioclema modzalevskaja Yaroshinskaya, 1990, Lioclema angulatum Yaroshinskaya, 1990, Amplexopora ramosa Yaroshinskaya, 1990. 


\section{Lochkovian}

Sibirca stage. - Age: early Lochkovian (Sibirka Formation). Trepostomid species present: Atactotoechus verus Yaroshinskaya, 1990, Eridotrypella ampla Yaroshinskaya, 1990, Lioclema modzalevskaja Yaroshinskaya, 1990, Lioclema angulatum Yaroshinskaya, 1990, Amplexopora ramosa Yaroshinskaya, 1990.

Remnyov stage. - Age: late Lochkovian (Remnyov Formation). Trepostomid species present: Lioclema multiacanthoporum Astrova, 1968, Lioclema netshlavense Astrova, 1964, Cyphotrypa variabile Mesentseva, 2008, Atactotoechus simplex L. Nekhoroshev, 1968.

\section{Pragian}

Pragian stage. - Age: Pragian (Yakushinkin Formation). Trepostomid species present: Lioclema subramosum U1rich \& Bassler, 1913.

\section{Emsian}

Early-Middle Salairka stage. - Age: early Emsian (lower and middle Salairka Formation). Trepostomid species present: Neotrematopora multi Mesentseva, 2000, Eridotrypa neocallosa Mesentseva, 2000, Eridotrypa grandialveolatum Mesentseva, 2000, Chondraulus salairicus Mesentseva, 2000, Cyphotrypa minor Mesentseva, 2000, Eostenopora notabilisica Mesentseva, 2003, Kysylschinipora sp. 1, Lioclema florea Modzalevskaya, 1965, Lioclema polymorpha Mesentseva, 1997, Neotrematopora spinula Mesentseva, 2000.

Late Salairka-Middle Shanda stage. - Age: middle Emsian (upper Salairka, Belovo, lower and middle Shanda formations). Trepostomid species present: Lioclema polymorpha Mesentseva, 1997, Lioclema lucida Mesentseva, 2000, Lioclema parvum Yaroshinskaya, 1974, Lioclema pseudobugusunica Mesentseva, 2000, Lioclema pseudogloria Mesentseva, 2000, Lioclema akarachica J. Udodov, 2004, Lioclema schandiensis Mesentseva, 2000, Paralioclema protea Mesentseva, 1997, Paralioclema magnumformis Mesentseva, 2000, Paralioclema volkovae Mesentseva,
1997, Neotrematopora spinula Mesentseva, 2000, Neotrematopora leptoclada Mesentseva, 2000, Neotrematopora salairiensis (Morozova, 1961), Neotrematopora crassiramosa Mesentseva, 2000, Neotrematopora celebrata (Yaroshinskaya, 1968), Neotrematopora vulgaris Mesentseva, 2000, Neotrematopora yolkini Mesentseva, 2000, Neotrematopora rudnoaltaica Mesentseva, 2000, Neotrematopora schebalinoensis Mesentseva, 2000, Eridotrypa kuzbassica Mesentseva, 2000, Eridotrypa nekhoroshevi Yaroshinskaya, 1968, Eridotrypa beloviense Mesentseva, 2000, Atactotoechus basarginensis Mesentseva, 2000, Anomalotoechus bublitschenkoi (Nekhoroshev, 1948), Leptotrypella gurievensis Astrova, 1968, Eostenopora tenuitubulosum Mesentseva, 2000, Eridotrypella pluristratosa J. Udodov, 2003.

Late Shanda stage. - Age: late Emsian (upper Shanda Formation). Trepostomid species present: Lioclema salairiense Morozova, 1961, Lioclema florea ampla Mesentseva, 1997, Paralioclema volkovae Mesentseva, 1997, Neotrematopora schebalinoensis Mesentseva, 2000, Neotrematopora pesterevensis J. Udodov, 2004, Neotrematopora pulchra J. Udodov, 2007, Minussina udodovi Mesentseva, 2000, Leptotrypa prima (Duncan, 1939).

\section{Eifelian}

Mamontovo stage. - Age: Eifelian (Mamontovo Formation). Trepostomid species present: Lioclema florea ampla Mesentseva, 1997, Neotrematopora pulchra J. Udodov, 2007, Neotrematopora eifeliensis J. Udodov, 2004, Raissiella fragilis J. Udodov, 2007, Eridotrypella distributa J. Udodov, 2003, Kysylschinipora sp. 2, Anomalotoechus typicus Duncan, 1939.

\section{Givetian}

Kerlegesh-Safonovo stage. - Age: early Givetian (Kerlegesh and Safonovo Formations). Trepostomid species present: Neotrematopora pulchra J. Udodov, 2007, Kysylschinipora sp. 3, Lioclema bascuscanensis J. Udodov, 2007, Lioclema rara J. Udodov, 2007, Leptotrypa spinosa J. Udodov, 2007, Eridotrypella bachatensis J. Udodov, 2007, Kysylschinipora sp. 4.

Figure 5. Emsian trepostomid bryozoans from the Salair (Gur'evsk town vicinity). • A, B - Cyphotrypa minor Mesentseva, 2000, sample B-829-12/6, holotype; A - tangential section, $\times 58$, B - longitudinal section, $\times 23$; Salairka Horizon, Emsian, Lower Devonian. $\bullet$ C-E - Leptotrypella gurievensis Astrova, 1968, sample B-829-16-/18; C - tangential section of macula, $\times 58$, D - tangential section, $\times 58$, E - longitudinal section, $\times 64$; Belovo Horizon, Emsian, Lower Devonian. • F, G - Eridotrypa neocallosa Mesentseva, 2000, sample B-829-11c/13, holotype; F - tangential section, $\times 76$, G - longitudinal section, $\times 60$; Salairka Horizon, Emsian, Lower Devonian. $\bullet$ H - Neotrematopora salairiensis (Morozova, 1961), sample B-829-15/51, longitudinal section, $\times$ 62; Salairka Horizon, Emsian, Lower Devonian. 


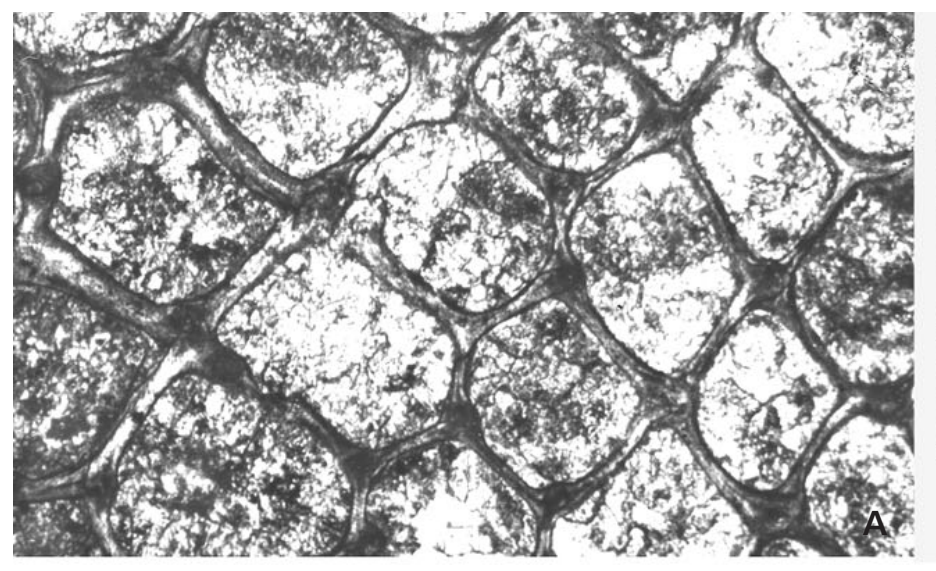

9 9

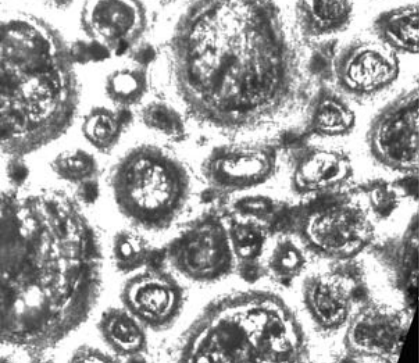

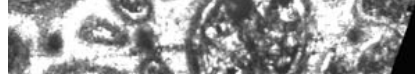
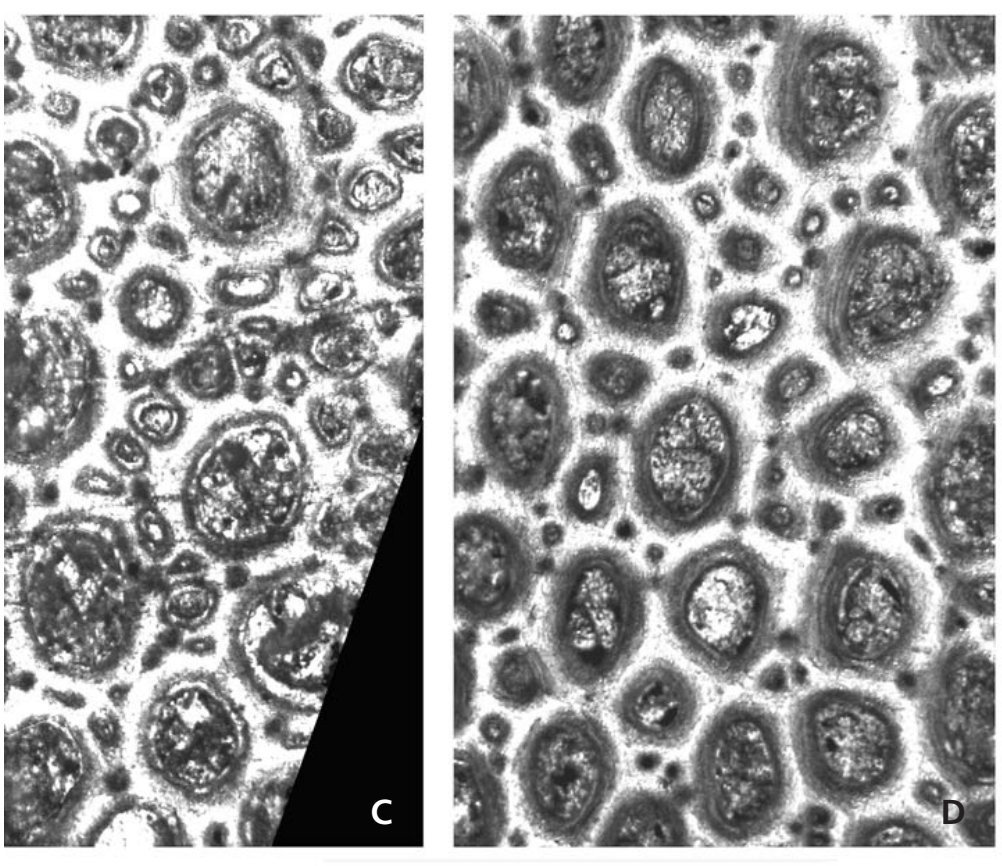

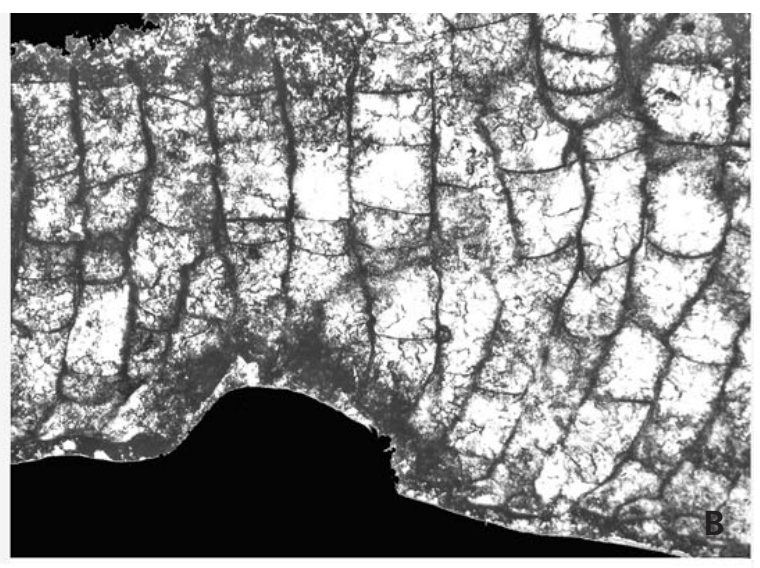

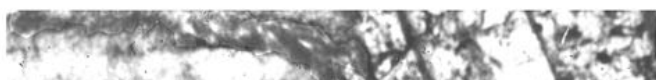
in 2 in

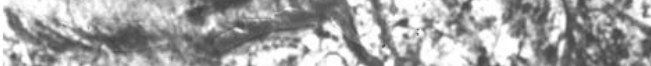
प्रा

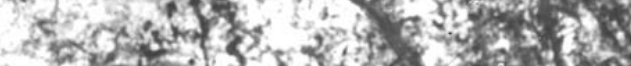

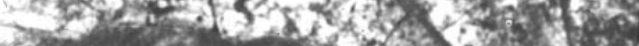

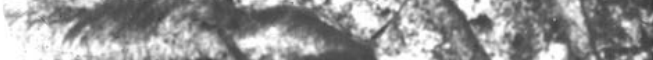

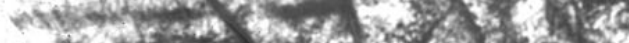

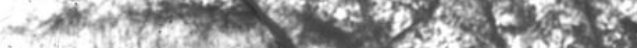

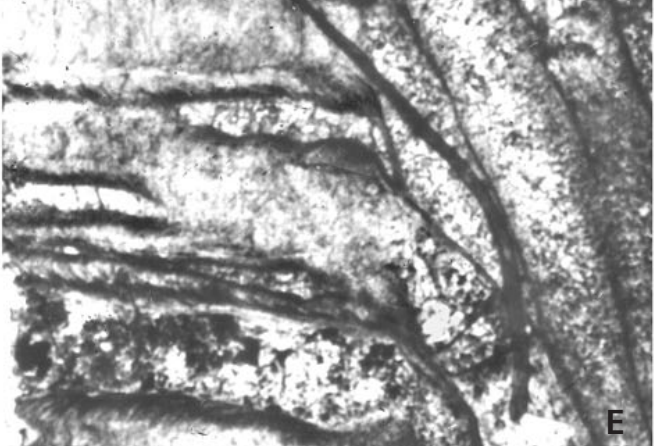

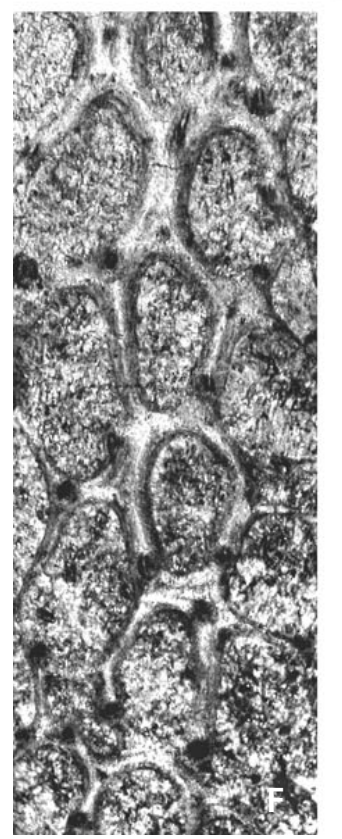
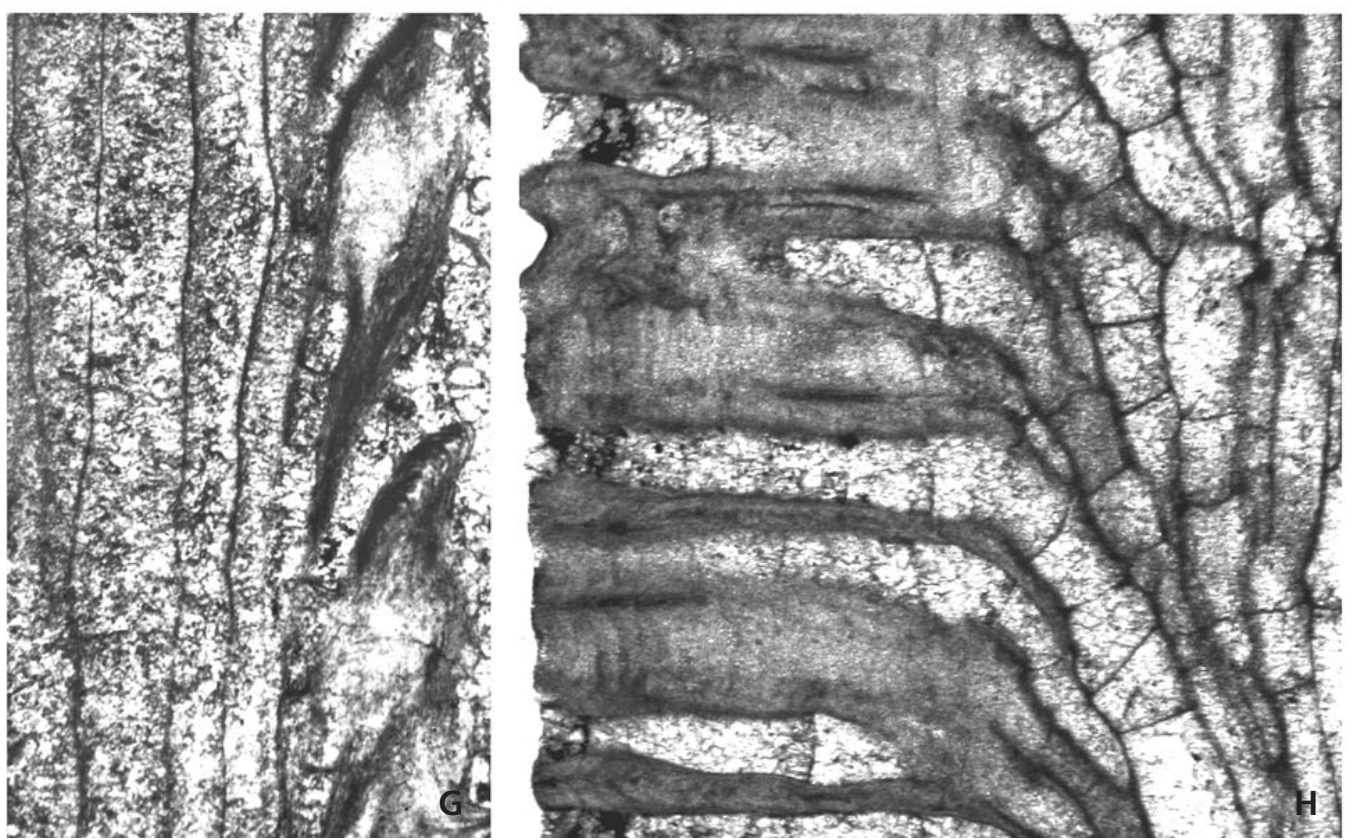

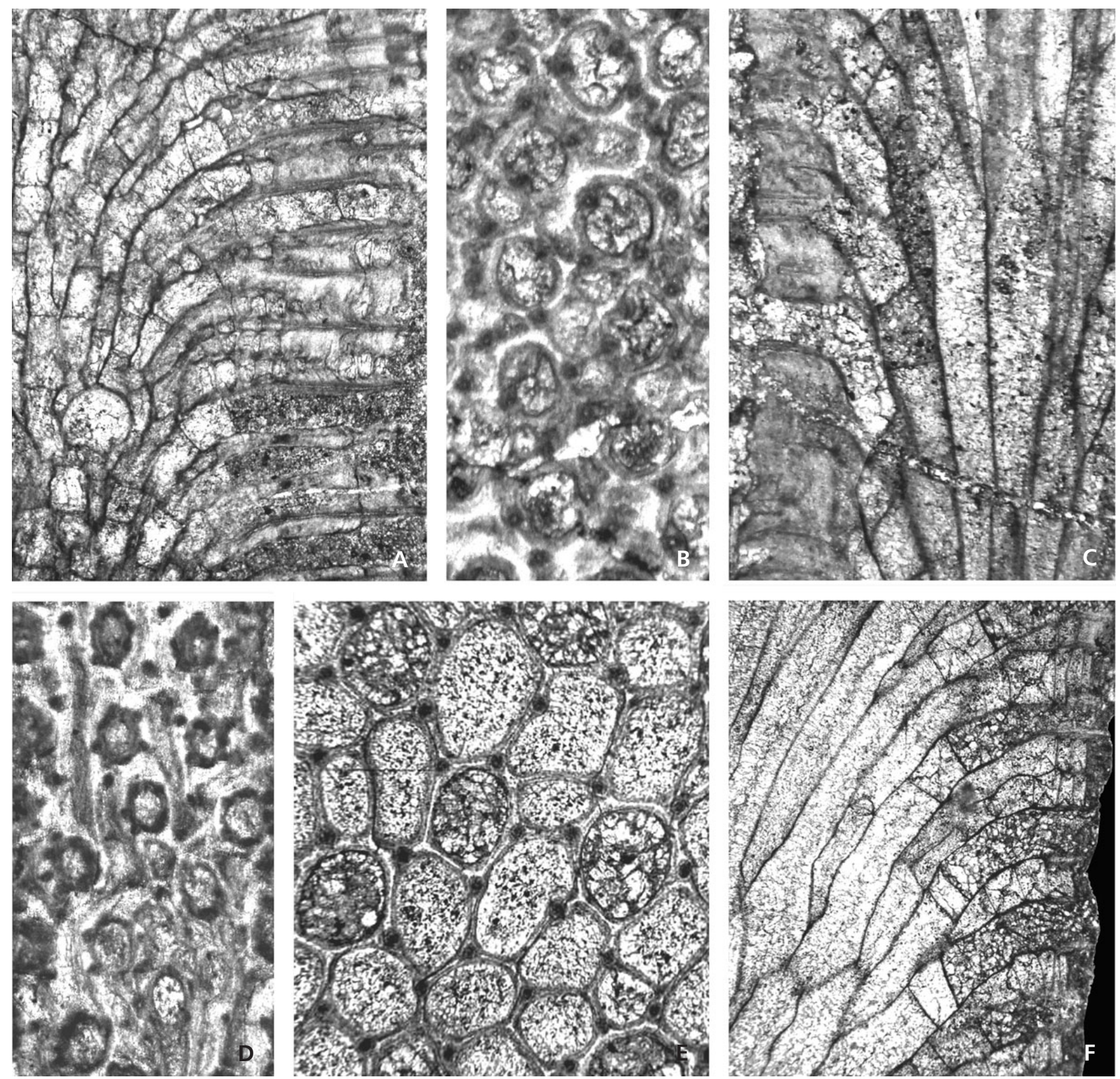

Figure 6. Emsian and Eifelian trepostomids from the Salair (Gur'evsk town vicinity). • A, B - Neotrematopora multi Mesentseva, 2000, sample Yo-837-1/1, holotype; A - longitudinal section, $\times 38$, B - tangential section, $\times 60$; Salairka Horizon, Emsian, Lower Devonian. $\bullet$ C, D - Raissiella fragilis J. Udodov, 2007, sample 29033/110, holotype; C - longitudinal section, × 70; D - tangential section, × 70; Mamontovo Horizon, Eifelian, Middle Devonian. $・ \mathrm{E}, \mathrm{F}-$ Lioclema schandiensis Mesentseva, 2000, sample Yo-828-1/2-2, holotype; $\mathrm{E}-$ tangential section, $\times 76, \mathrm{~F}-$ longitudinal section, $\times 32$; Shanda Horizon, Emsian, Lower Devonian.

Mazalovskiy Kitat stage. - Age: late Givetian (Alchedat and Izyly formations). Trepostomid species present: Lioclema bugusunicum Nekhoroshev, 1948, Lioclema polenovi Nekhoroshev, 1948, Lioclema mirum Morozova, 1961, Lioclema multum Volkova, 1974, Lioclema celebratum Morozova, 1961, Lioclema maculatum Nekhoroshev, 1948, Lioclema occidens (Hall \& Whitfield, 1873), Paralioclema vulgatum Morozova, 1961, Monotrypa usitata Volkova, 1974, Neotrematopora typica Morozova,
1961, Neotrematopora tschuensis (Nekhoroshev, 1948), Neotrematopora plena Volkova, 1974, Neotrematopora koksairiensis Volkova, 1974, Neotrematopora vasilievskiji (Schoenmann, 1926), Minussina kitatensis Morozova, 1961, Cyphotrypa minuscula Volkova, 1974, Atactotoechus solidus Volkova, 1974, Anomalotoechus crassimuralis (Volkova, 1974), Anomalotoechus corrugatus (Nekhoroshev, 1948), Anomalotoechus kisilschinicus (Nekhoroshev, 1948), Leptotrypella tenuis Volkova, 1974, 

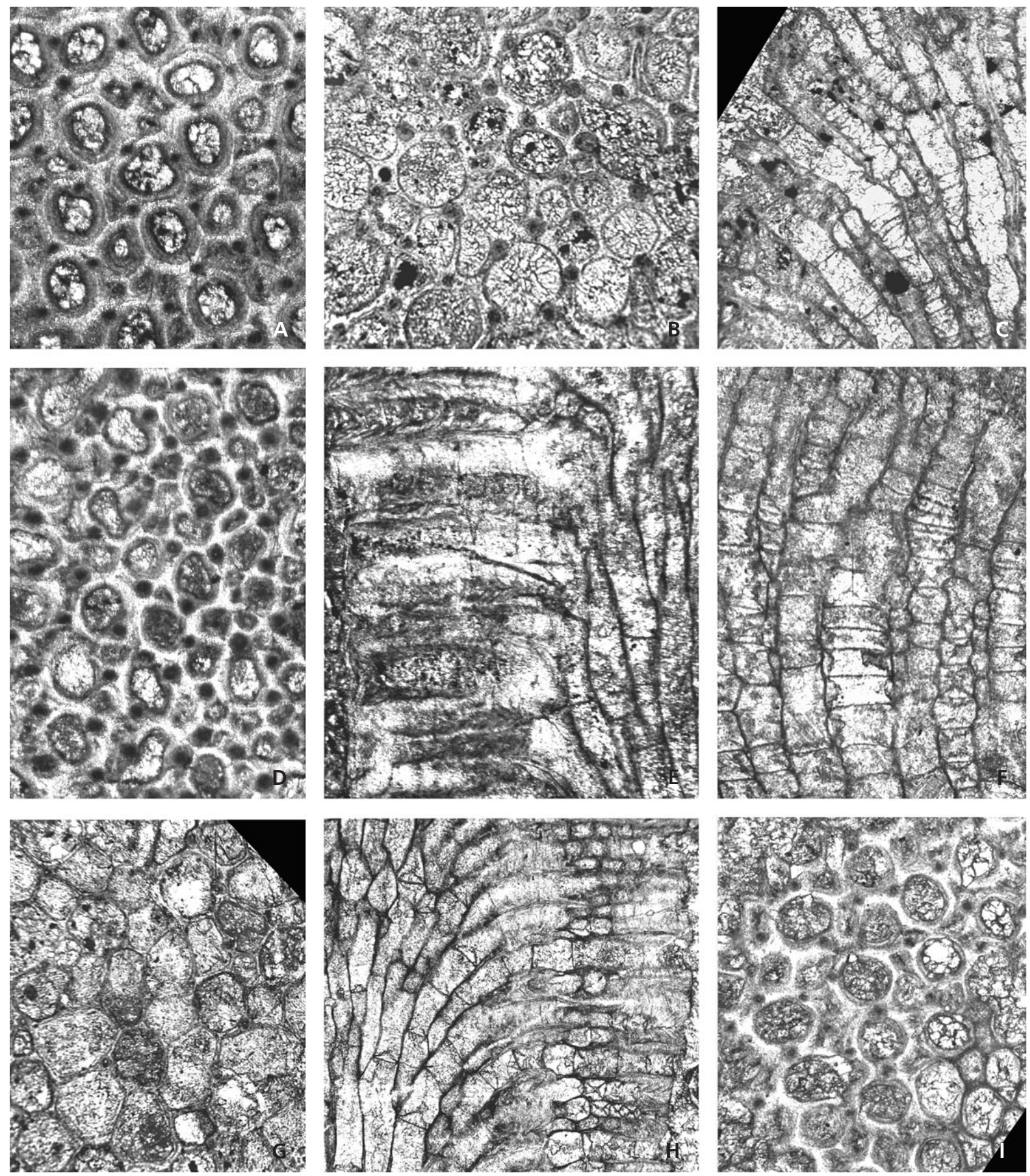

Figure 7. Emsian trepostomids from the Salair (Gur'evsk town vicinity, Novo-Pesteryovo Village, right bank of Kara-Chumysh River). - A - Neotrematopora salairiensis (Morozova, 1961), sample B-829-15/51, tangential section, $\times$ 58; Salairka Horizon, Emsian, Lower Devonian. - B, C - Paralioclema magnumformis Mesentseva, 2000, sample Ya-854-3/10, holotype; B - tangential section, $\times 54$, C - longitudinal section, $\times$ 30; Shanda Horizon, Emsian, Lower Devonian. • D, E - Neotrematopora yolkini Mesentseva, 2000, sample Yo-828-1/1-199, holotype; D - tangential section, $\times$ 46; E - longitudinal section, $\times 57$; Shanda Horizon, Emsian, Lower Devonian. $\bullet$ F, G - Lioclema salairiense Morozova, 1961, sample 25030/1; F - longitudinal section, $\times 38$; G - tangential section, $\times 50$; Shanda Horizon, Emsian, Lower Devonian. $\bullet$ H, I - Neotrematopora pesterevensis J. Udodov, 2004, sample B-8323-3/6, holotype; $\mathrm{H}-$ longitudinal section, $\times 36$, I - tangential section, $\times 52$; Shanda Horizon, Emsian, Lower Devonian. 
Leptotrypella protea Volkova, 1974, Dyscritella devonica Volkova, 1968, Petalotrypa perforata Nekhoroshev, 1948, Eridotrypella grandis (Volkova, 1974), Eridotrypella devonica (Nekhoroshev, 1948), Eridotrypella valentinae Morozova, 1961, Eridotrypella angusta Volkova, 1974, Eridotrypella perrara Volkova, 1974, Eridotrypella rara Volkova, 1974, Eridotrypella instabilis Volkova, 1974, Kysylschinipora orbis Volkova, 1974, Kysylschinipora nekhoroschevi Volkova, 1974, Anomalotoechus ramosus (Morozova, 1959), Lioclema yakovlevi (Schoenmann, 1926), Lioclema heitaiense Yang, 1956.

\section{Frasnian}

Vassino stage. - Age: early Frasnian (Vassino Formation). Trepostomid species present: Lioclema yakovlevi (Schoenmann, 1926), Lioclema heitaiense Yang, 1956, Lioclema vassinense Morozova, 1961, Lioclema tschuyaense Volkova, 1969, Lioclema plicatile Volkova, 1969, Lioclema incrustans Nekhoroshev, 1948, Lioclema editum Morozova, 1961, Paralioclema ninae Morozova, 1961, Paralioclema multiforme Volkova, 1974, Minussina atypica Volkova, 1974, Minussina grandis Morozova, 1961, Anomalotoechus ramosus (Morozova, 1959), Cyphotrypa definita Morozova, 1961, Leptotrypa rhombocella Morozova, 1961, Anomalotoechus yayaensis Morozova, 1957, Anomalotoechus insuetus (Morozova, 1959), Atactotoechus belskayae Morozova, 1957, Leptotrypella aperta Morozova, 1958, L. inaudita Morozova, 1961, Eridotrypella conspersa (Volkova, 1974).

Solomino stage. - Age: late Frasnian (Solomino Formation). Trepostomid species present: Lioclema editum Morozova, 1961, Minussina akkayensis Volkova, 1974, Anomalotoechus bifoliatus (Morozova, 1959), Eridotrypella ornamentata (Morozova, 1958), Eridotrypella justa Morozova, 1961, Eridotrypella granulosa (Morozova, 1955), Schulgina nesterenkoae Morozova, 1957.

\section{Famenian}

Peshcherka stage. - Age: early Famennian (Peshcherka Formation). Trepostomid species present: Lioclema ivanovae Morozova, 1961, Lioclema numerosum Morozova,
1961, Neotrematopora majuscula (Volkova, 1974), Minussina akkayensis Volkova, 1974, Anomalotoechus laminaris Tolokonnikova, 2006, Microcampylus daedaleus Tolokonnikova, 2006, Crustopora devonica Tolokonnikova, 2006.

Podonino stage. - Age: middle Famennian (Podonino Formation). Trepostomid species present: Minussina akkayensis Volkova, 1974, Lioclema ramosum Nekhoroshev, 1948, Anomalotoechus proprius Tolokonnikova, 2006, Eridocampylus striatum Tolokonnikova, 2006, Eridocampylus rotundatum Tolokonnikova, 2006.

Abyshevo stage. - Age: late Famennian (Abyshevo Formation). Trepostomid species present: Lioclema podunskense Trizna, 1958, Lioclema textilum Trizna, 1958, Tabuliporella vesca Trizna, 1958, Tabuliporella risilla Trizna, 1958.

\section{Carboniferous, Tournaisian}

Early Tournaisian stage. - Age: early Carboniferous (Taydon Formation). Trepostomid species present: Tabuliporella mosjuchensis Trizna, 1958, Petalotrypa kossmati $\mathrm{Ni-}$ kiforova, 1933.

\section{Results}

Boundaries of the trepostomid biozones are considered as times of evolutionary innovations. The number of trepostomid species that pass through such boundaries usually does not exceed two or three, except for four, the LudlowPřídolian, Přídolí-early Lochkovian, Middle-Late Shanda, Late Taydon-Fominskoe, where it is four to six species. The comparatively large number of species passing through the Přídolian-early Lochkovian boundary, apparently reflects insufficient study of this interval, which has meant that the boundary has previously been placed at different stratigraphical levels.

The results obtained allow the use of Devonian trepostomids from the SAR for the correlation of horizons. In some cases more detailed correlations are also possible. In particular, trepostomids now allow correlation of the Upper Shanda Subhorizon sequences within the SAR. The

Figure 8. Eifelian and Early Givetian trepostomids from the Salair (Gur'evsk and Prokop'evsk towns vicinity). • A, B - Eridotrypella distributa J. Udodov, 2003, sample 29033/30, holotype; A - tangential section, × 66, B - longitudinal section, × 67; Mamontovo Horizon, Eifelian, Middle Devonian. $•$ C, D - Leptotrypa spinosa J. Udodov, 2007, sample M-01-50/37, holotype; C - longitudinal section, $\times 56$, D - tangential section, $\times 52$; Safonovo Horizon, lower part of Givetian, Middle Devonian. $\bullet$ E, F - Kysylschinipora sp. 2, sample 29030/28, holotype; E - tangential section, $\times 56$, F - longitudinal section, $\times 52$; Mamontovo Horizon, Eifelian, Middle Devonian. $\bullet$ G-I - Kysylschinipora sp. 3., sample 29008/2, holotype; G - tangential section, $\times 66, \mathrm{H}-$ longitudinal section, $\times 56, \mathrm{I}-$ tangential section of macula, $\times 52$; Safonovo Horizon, lower part of Givetian, Middle Devonian. 


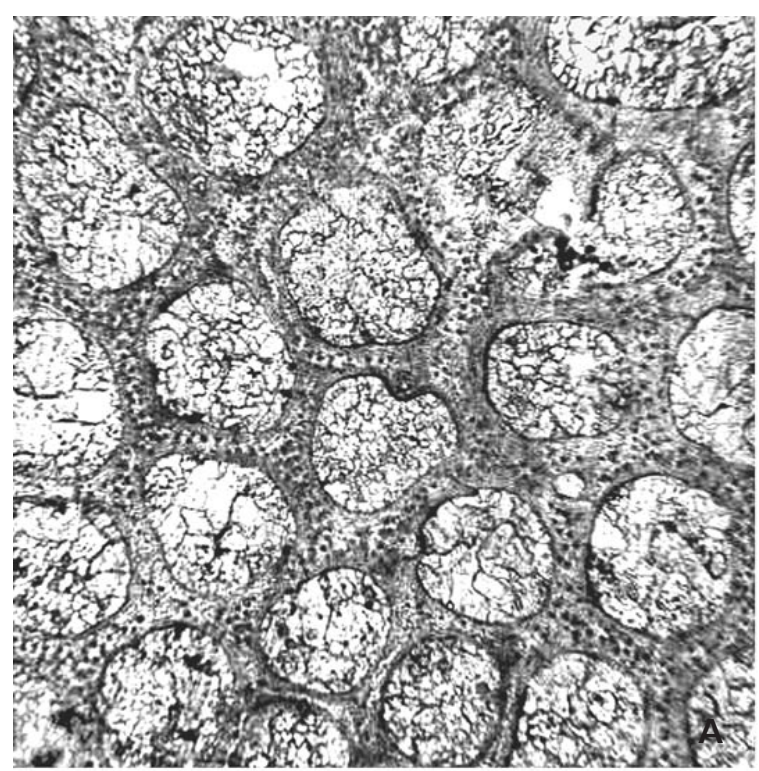

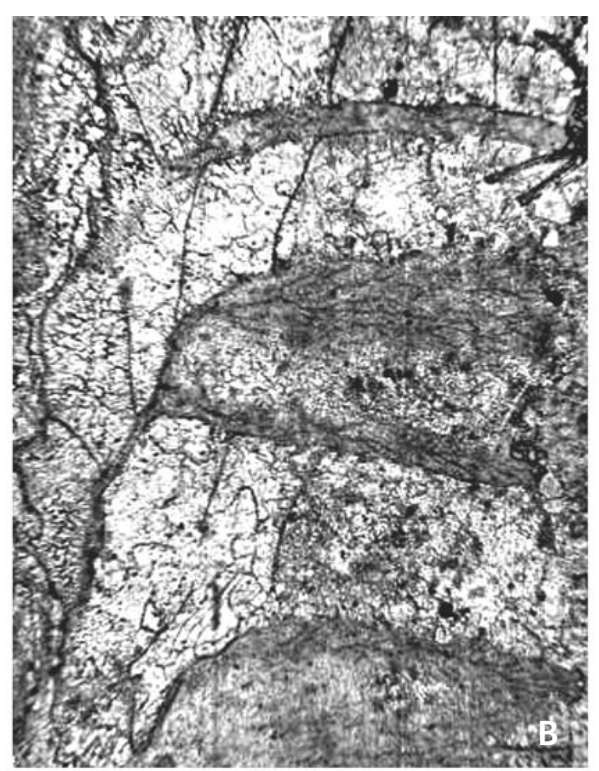

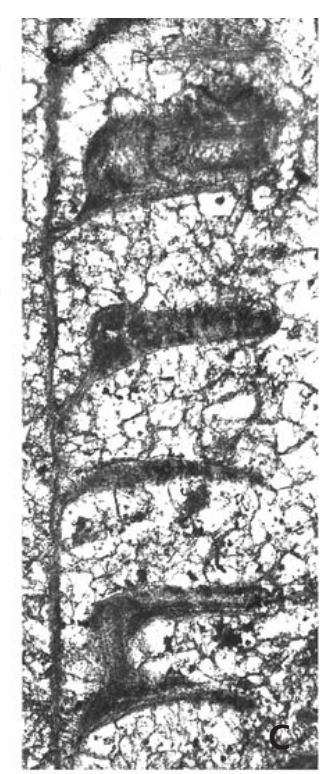

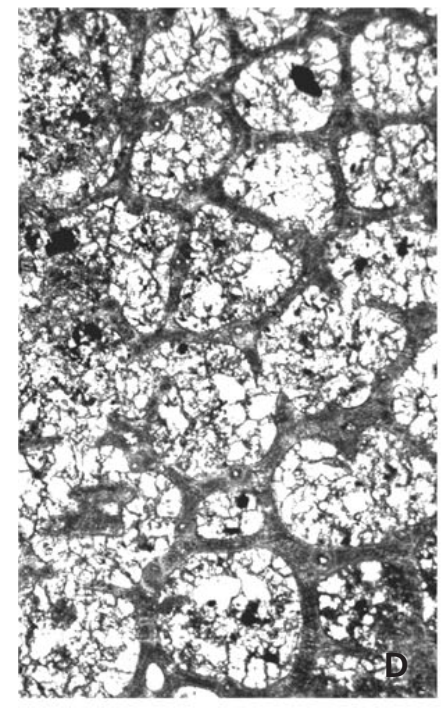

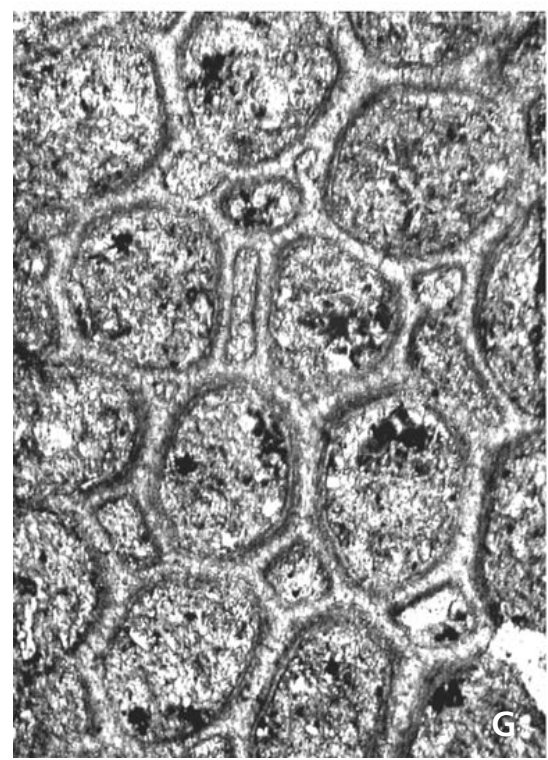

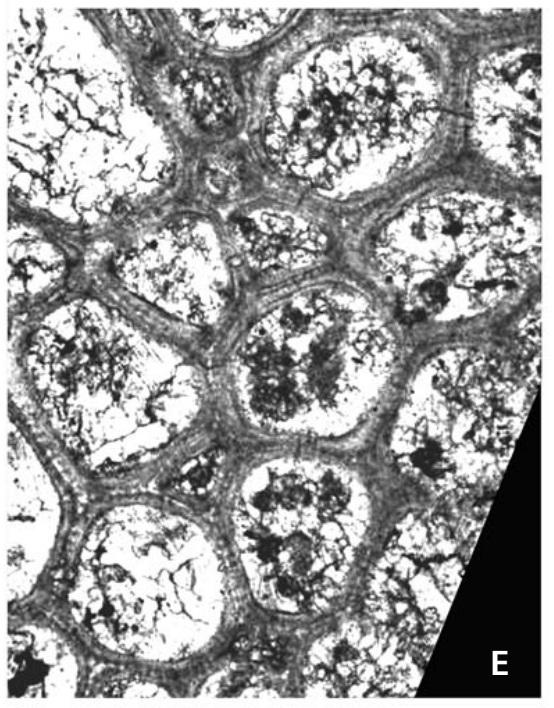

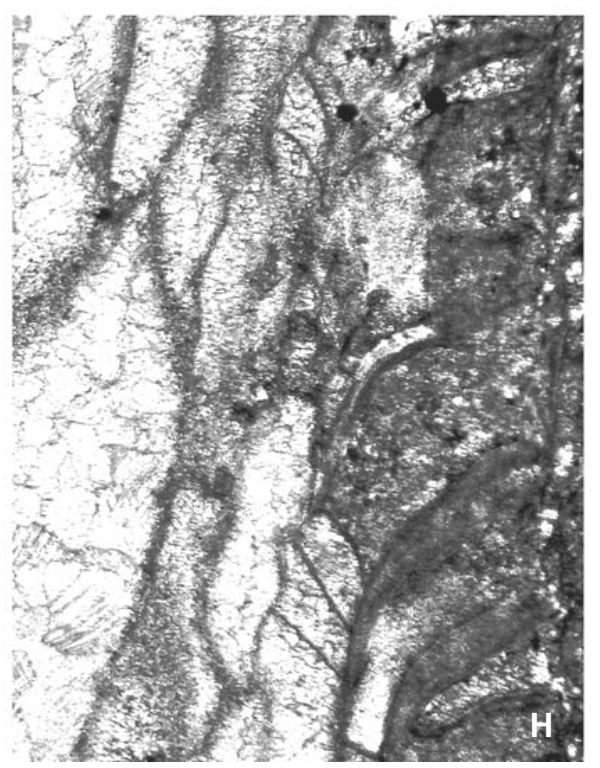

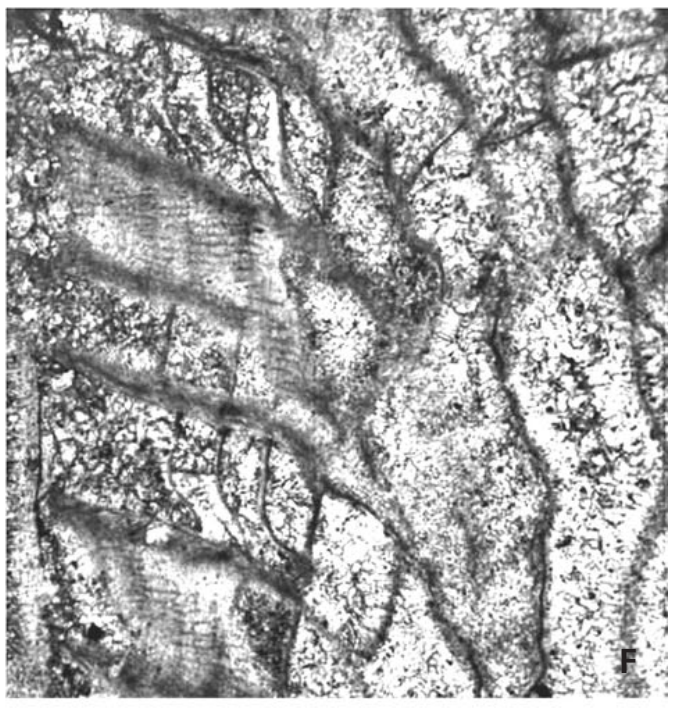

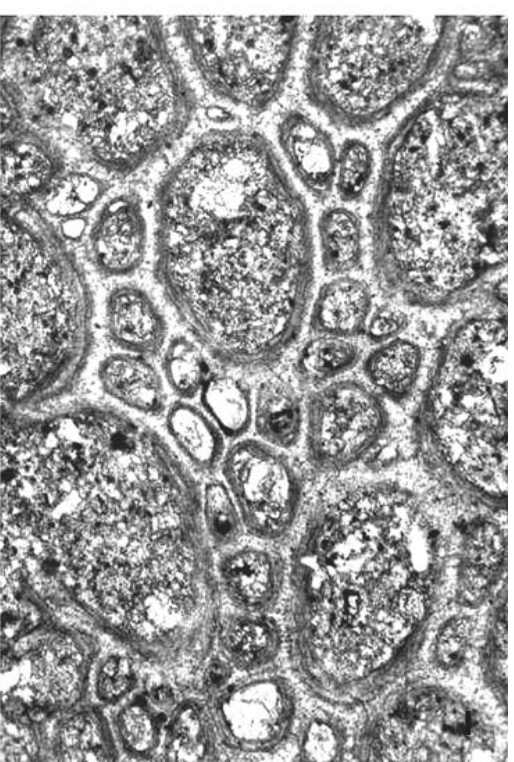


species composition of the Lower and Upper Salairka trepostomid faunas are useful for correlation. Along with other marine faunal groups the trepostomids have been included in a new version of the regional stratigraphic chart for the Devonian of the ASFA that is under preparation.

Diverse Devonian trepostomid species have been obtained from the SAR (Fig. 3). The late Lochkovian-Pragian (Peetz-Maly Bachat age), early Givetian (KerlegeshSafonovo age) and late Famennian-Early Tournaisian (Abyshevo-Taydon age) intervals are characterized by the lowest species diversities (1-8 species). Maximum species diversities (19-36 species) were observed in the Přídolian, Middle Emsian (Late Salairka-Middle Shanda age), late Givetian (Mazalovsky Kitat age), Early Frasnian (Vassino age) and late Tournaisian (Fominskoe age). Thus, during the late Silurian-Tournaisian, alternations of times of the low and high trepostomid species diversity occurred, producing a cyclicity. Three cycles can be recognized: Př́dolian-Pragian, Emsian-early Givetian and late Givetian-early Tournaisian with durations of 16, 19 and 30 million years, respectively. In the second half of the Tournaisian a fourth Early-mid-Carboniferous cycle began. The start of each cycle is characterized by high species diversity, the end by low diversity. Three of the cycles (Emsian-early Givetian, late Givetian-early Tournaisian and early-middle Carboniferous) coincide with cycles of transgression-regression such that phases of maximum diversity correspond to phases of transgression (middle Emsian, late Givetian, late Tournaisian), and those of minimum diversity to epochs of regression (early Givetian, late Famennian, late Viséan). The only exception is the late Lochkovian-Pragian, which coincided with a transgression, but is characterized by low species diversity. The reasons for this anomaly remain unclear. However, the prevailing facies during the late Lochkovian-Pragian transgression provide a possible explanation. During this time coral reefs were formed within the Salair paleobasin with rare trepostomids. Back reef facies often possessed abundant trepostomids, but their species diversity was low. Lagoons occurring in the Altai are characterized by the broadest distribution but of only one species. In particular, the Pragian sequences in the vicinity of Kur'ya Village contain only Lioclema subramosum Ulrich \& Bassler, 1913.

\section{Discussion \& Conclusions}

The dynamics of trepostomid species diversity in the SAR can be compared with data on the global diversity of this group at generic level for the Devonian published by Goryunova et al. (2004). To make this comparison the diagram for trepostomid genera was made for the SAR (Fig. 3, curve $a, b)$.
The patterns for the SAR are very similar at species and genus levels. However, they contrast with Goryunova et al.'s (2004) global pattern (Fig. 3, curve c), which is interpreted as caused by the fact that the dynamics at regional and global levels were controlled by different factors. The global pattern probably reflects the transgression-regression cycles driven by global eustatic sea-level fluctuations. The diversity dynamics of the trepostomids in the SAR reflects the transgression-regression at a regional level. During the Devonian the territory of the SAR experienced high levels of tectonic activity. Transgression-regression cycles in this region were driven mostly by tectonic movement and only by global eustatic sea-level fluctuations to a small degree.

The stratigraphic range of trepostomid species has been determined on the basis of modern data on the duration of geological epochs from the International Geochronological Scale (Gradstein et al. 2004) and new data on trepostomid distribution in upper Silurian-Tournaisian sections in the SAR (Fig. 2). In total, ranges of 151 species have been established (Fig. 4).

Time ranges of $40 \%$ of trepostomid species do not exceed 3 million years; over $60 \%$ of species existed for less than 4 million years; over $70 \%$ of species for less than 6 million years; about $25 \%$ of species are characterized by significantly longer time ranges (6-10 million years). These latter ranges are similar to brachiopods and corals, supporting significantly longer time ranges (6-10 million years). This similarity to brachiopods and corals supports the significant biostratigraphical value of trepostomids.

\section{Acknowledgements}

The author would like to thank O.T. Obut for help with English translation; V.R. Savitsky for taking photographs of thin sections with bryozoans and preparation of photoplates. This is a contribution to UNESCO-IUGS IGCP project 499 "Devonian land-sea interaction: evolution of ecosystems and climate".

\section{References}

ASTROVA, G.G. 1959. Siluriiske mshanki tzentralnoi i zapadnoi Tuvui. Trudy Paleontologischeskogo Instituta Akademiya Nauk SSSR 79, 1-74. [in Russian]

AstrovA, G.G. 1964. A new order of Paleozoic Bryozoa. (O novom otryade paleozojskikh mshanok.) Paleontologicheskii Zhurnal 1964(2), 22-31. [in Russian]

AstrovA, G.G. 1965. Morphologiya, istoriya razvitiya i sistema ordoviksikh i siluriyskikh mshanok. (Morphology, history of development, and systematics of Ordovician and Silurian Bryozoa). Trudy Paleontologischeskogo Instituta Akademiya Nauk SSSR 106, 1-432. 
Astrova, G.G. \& YAROShinskayA, A.M. 1968. Rannedevonskie i eifelskie mshanki Salaira i Gornogo Altaya. Trudy Tartuskogo gosudarstvennogo universiteta 202, 47-62. [in Russian]

BakhareV, N.K., Sennikov, N.V., Yolkin, E.A. \& IzOKH, N.G. 2004. Key Devonian sections from Rudny Altai, Salair and Kuznetsk Basin regions. 104 pp. Publishing House of SB RAS, Novosibirsk. [in Russian]

CherePnina, S.K. \& YAROShInSKAYA, A.M. 1974. New taxa of early Devonian and Eifelian rugose corals and bryozoans. Trudy Tomskogo Gosudarstvennogo Universiteta 227, 154-168. [in Russian]

DunCAN, H. 1939. Trepostomatous Bryozoa from the Traverse Group of Michigan. Contributions from the Museum of Paleontology, University of Michigan 5, 171-270.

HALL, J. \& WHITFIELD, R.P. 1873. Descriptions of new species of fossils from the Devonian rocks of Iowa. New York State Cabinet of Natural History, Annual Report 23, 223-239.

Goryunova, R.V., MARKov, A.V. \& NAIMARK, E.B. 2004. Evolution and biogeography of Paleozoic bryozoans: the results of quantitative analysis. $182 \mathrm{pp}$. GEOS, Moscow. [in Russian]

Gradstein, F.M., OGG, J.G., SMith, A.G., BleEKer, W. \& LOURENS, L.J. 2004. A new Geologic time scale, with special reference to Precambrian and Neogene. Episodes 27(2), 83-100.

GUTAK, J.M. 2006. Razrez sredne-verchnedevonskich otlozheny v doline r. Kyzylshin. Priroda i ekonomika Kuzbassa 10, 29-34. [in Russian]

KopaJEVICH, G.V. 1968. On bryozoan new genus, Family Rhinidictyidae in Silurian of Estonia. Byulleten' Moskovskogo Obshchestva Ispytatelei Prirody, Otdel Geologicheskii 43(3), 127-129. [in Russian]

Mesentseva, O.P. 1997. Novye taksony rodov Lioclema i Paralioclema (Bryozoa) iz emsskich otlozhenii Salaira i Gornogo Altaya. Voprosy geologii i paleontologii Sibiri, XX, 121-128. [in Russian]

Mesentseva, O.P. 2000. New Emsian (Lower Devonian) species of the genus Neotrematopora (bryozoans) from the Salair and Gorny Altai (southern West Siberia). News of paleontology and stratigraphy. Supplement to Russian Geology and geophysics 41(2-3), 61-71. [in Russian]

MesentseVA, O.P. \& UdOdOV, J.V. 2000. Rasprostranenie trepostomid (mshanki) V nizhne-srednedevonskikh otlozheniyakh yugo-zapadnoi periferii Kuzbassa. Priroda i ekonomika Kuzbassa 8, 37-45. [in Russian]

MesentseVA, O.P. \& Udodov, J.V. 2003. Ob ob'eme rodov Eostenopora Duncan i Eridotrypella Duncan. Mshanki Zemnogo shara. Mezhdunarodnyi sbornik nauchnykh statei, 73-92. [in Russian]

MESENTSEVA, O.P. \& UdODOV, V.P. 2007. Emsskie formacii $i$ paleotektonicheskoe raionirovanie territorii Gornogo Altaya. 103 pp. KuzGPA, Novokuznetsk. [in Russian]

Mesentseva, O.P., Udodov, V.P. \& Udodov, J.V. 2007. Rasprostranenie trepostomid (mshanki) v nizhnezhivetskikh otlozheniyakh Salaira. Priroda i ekonomika Kuzbassa 11, 38-49. [in Russian]
ModzalevsKayA, E.A. 1978. Bryozoa complexes from the Chergak series in Tuva. Ezhegodnik Vsesoyuznogo Paleontologicheskogo Obshchestva 21, 119-147. [in Russian]

ModZAlEVSKAYA, E.A. 1979. Some Silurian Bryozoa from Tuva. Ezhegodnik Vsesoyuznogo Paleontologicheskogo Obshchestva 22, 63-93. [in Russian]

ModZALEVSKAYA, E.A. \& NeKHOROSHEV, V.P. 1965. Early Devonian Bryozoa of the Upper Amur region. Ezhegodnik Vsesoyuznogo Paleontologicheskogo Obshchestva 17, 115-137. [in Russian]

MorozovA, I.P. 1961. Devonskie mshanki Minusinskikh $i$ Kuznetskoi kotlovin. 172 pp. AN SSSP, Moscow. [in Russian]

NeKHOROSHEV, V.P. 1948. Devonskie mshanki Altaya. 172 pp. AN SSSP, Moscow. [in Russian]

NEKHOROSHEV, V.P. 1956. Rannekamennougolnye mshanki Altaya i Sibiri. 419 pp. Gosgeoltekhizdat, Moscow. [in Russian]

NiKIFOROVA, A.I. 1933. Middle Carboniferous Bryozoa of the Donetz Basin. Trudy Vsesoyuznogo Geologo-razvedochnogo ob'edinenya 237, 1-45. [in Russian]

Pushin, V.I., NekhorosheVa, L.V., KopaeVich, G.V. \& YAROSHINSKAYA, A.M. 1990. Przhidol'skie mshanki SSSR. 125 pp. Nauka, Moscow. [in Russian]

RZONSNITSKAYA, M.A. 1968. Biostratigraphiya devona okrain Kuznetskogo basseina. 287 pp. Nedra, Leningrad. [in Russian]

SchoenmanN, Y. M. 1926. The Middle Devonian Trepostomata from the district of Minussinsk, Enissey gov., Siberia. Izvestiya Geologiceskogo komissii (Leningrad) 44(10), 917-935. [in Russian]

STEPANOV, S.A., MiRONOVA, N.V., ASTASHKINA, V.F., LOPUSHINSKAJA, T.V., CHEREPNINA, S.K. \& JAROShINSKAYA, A.M. 1972. Devonskie otlozheniya okrestnostei s. Sibiryachihi (bassein r. Anuya, Gornyi Altai). Trudy Sibirskogo nauchno-issledovatel'skogo instituta geologii, geofiziki i mineral'nogo syr'ya Ministerstva geologii i okhrany nedr SSSR 146, 93-103. [in Russian]

sine 1992. Tipovye razrezy pogranichnykh otlozhenii srednego $i$ verhnego devona, franskogo i famenskogo yarusov okrain Kuznetskogo basseina. 185 pp. SNIIGGiMS Press, Novosibirsk. [in Russian]

TOLOKONNIKOVA, Z.A. 2006. Novye famenskie mshanki yayapetropavlovskogo razreza (Kuzbass). Priroda i ekonomika Kuzbassa 10(1), 45-48. [in Russian]

TRIZNA, V.B. 1958. Rannekamennougolnye mshanki Kuznetskoi kotloviny. 434 pp. Gostoptekhizdat, Leninigrad. [in Russian]

UdODOV, J.V. 2004. Ranne-srednedevonskie trepostomidy (mshanki) Salaira $i$ ikh stratigraficheskoe znachenie. Avtorepherat dissertacii. 24 pp. Tomsk. [in Russian]

Udodov, V.P., GutaK, J.M., Mesentseva, O.P, SELIVERSTOVA, M.M. \& ZADEREI, V.S. 1987. Ob etalonnom razreze vassinskogo gorizonta yuzhnoi okrainy Kuzbassa. Priroda $i$ ekonomika Kuzbassa 5, 51-53. [in Russian]

ULRICH, E.O. \& BASSLER, R.S. 1913. Systematic paleontology of the Lower Devonian deposits of Maryland; Bryozoa. Maryland Geological Survey, Lower Devonian, 259-290. 
VolKova, K.N. 1974. Devonskie mshanki Ygo-Vostochnogo Altaya. 181 pp. AN SSSR, Moscow. [in Russian]

YANG, JiNGZHI 1956. The Middle Devonian Bryozoa from the Heitai Formation of Mishan County, Kirin Province. Scientia Sinica 5, 763-793.

YAROSHINSKAYA, A.M. 1997. Osobennosti przhidol'skich mshanok Gornogo Altay i Tuvy, 269-272. In PoDOBINA, V.M., SAVINA, N.I., KuZNETSOVA, K.I. \& MuZYLEV, N.G. (eds) Biostratigrafiya i mikroorganizmy fanerozoya Evrazii. Geos, Moscow. [in Russian]

YOLKIN, E.A. 1968. Trilobity (dehenellidy) $i$ stratigraphiya nizhnego i srednego devona yuga Zapadnoi Sibiri. 154 pp. Nauka. Novosibirsk. [in Russian]

YolKin, E.A., BAKHAREV, N.K., GRATSIANOVA, R.T., ZHELTONOGOVA, V.A., IZOKH, N.G., JAZIKOV, A.J., VALIUKEVICIUS, J.J., VyUShKova, L.V. \& TALimaA, V.N. 1986. Stratotipicheskie razrezy nizhnego i srednego devona Salaira. Telengitskii nadgorizont: terrigenno-karbonatnye facii. 143 pp. Akademija Nauk SSSR, Sibirskoie otdelenie Institut geologii i geofisiki, Stuttgart. [in Russian]

YOLKIN, E.A., BAKHAREV, N.K., GRATSIANOVA, R.T., ZHELTONOGOVA, V.A., IZOKH, N.G., JAZIKOV, A.J., VYUSHKOVA, L.V., Mezentseva, O.P., Petrosjan, N.M. \& TimoKHINA, I.G. 1987. Stratotipicheskie razrezy nizhnego $i$ srednego devona Salaira. Telengitskii nadgorizont: karbonatnye facii. 194 pp. Akademija Nauk SSSR, Sibirskoie otdelenie Institut geologii i geofisiki, Novosibirsk. [in Russian]

YOLKIN, E.A., BAKHAREV, N.K., IZOKH, N.G., GRATSIANOVA, R.T., KIPRIYANOVA, T.P. \& OBUT, O.T. 2005. Devonian sequences of Salair, Rudny i Gorny Altai. 82 pp. SB RAS, "Geo" Branch, Novosibirsk.

Yolkin, E.A., GRATSIANOVA, R.T., IZOKH, N.G., YAZIKOV, A.Y. \& BAKHAREV, N.K. 1997. Devonian sea-level fluctuations on the south-western margin of the Siberian continent. Courier Forschungsinstitut Senckenberg 199, 83-98. 